\title{
KEEPING THE BEAT AS A MEASURE OF SPECIFIC LEARNING DIFFICULTIES USING MIDI CONTROLLER
}

\author{
Eva Gyarmathy ${ }^{1}$, Csaba Kertesz ${ }^{2}$, Bertalan Forstner ${ }^{3}$ \\ ${ }^{1}$ Institute of Cognitive Neuroscience and Psychology of the Research Centre for Natural \\ Sciences, Hungarian Academy of Sciences (HUNGARY) \\ ${ }^{2}$ KRE - Institute of Psychology (HUNGARY) \\ ${ }^{3}$ Budapest University of Technology and Economics (HUNGARY)
}

\begin{abstract}
The aim of our research is to work out a testing program to differentiate the typical and atypical sensory motor development. In this recent study we examined children with and without specific learning difficulties using midi-controller, a small percussion-like device as the tool for the testing.

In a previous study we found age differences in children in the beat-keeping accuracy using the midicontroller, which indicates that this variable is crucial and indicative in the maturation of the brain and our test device is appropriate for the assessment (Kertesz, 2016).

In our recent research the test results show significant differences between the beat keeping accuracy of children with and without specific learning difficulties, which means that the device can be used as an aid in the early identification of specific learning difficulties, and can be developed for a training device, too.
\end{abstract}

Keywords: sensory motor synchronization, rhythm perception, atypical development, learning difficulties, digital test devices.

\section{INTRODUCTION}

\subsection{Atypical development}

Dyslexia, dyspraxia, dyscalculia, ADD, ADHD and autism are considered as being separate disorders still. However tremendous research data prove the co-morbidity, and that a child may often exhibit more than one of these complex syndromes.

Research evidence suggests that motor planning and sequencing, rhythm and timing are relevant to ADHD and dyslexia as well as to autism spectrum disorders. Deficits in inhibition and executive functions, which involve the regulation and sequencing are important part of the ADHD. Several data shows relationship between attention, motor regulation, inhibition, speed, rhythm and coordination (Schonfeld, et al, 1989; Barkley, 1997; Piek et al, 1999). Similar results are proved at dyslexic children (Denckla, et al, 1985) and children with autism (Greenspan, Wieder, 1999).

Dyscalculia and dyslexia have been shown to be combined with attention-deficit hyperactivity disorder at a rate of $26 \%$ and $33 \%$. In addition, $17 \%$ of children with dyscalculia also showed considerable deficits in reading (Gross-Tsur, Manor, Shalev, 1996; Habib, 2000, Mayes, Calhoun, 2006; Shaywitz, Shaywitz, 1988). A variety of executive functions deficits have been found in dyslexia (Welsh et al., 1991; Reiter, et al 2005), in ADHD (e.g. Sergeant, et al, 2002) and in autism (e.g. Hill, 2004).

Pauc (2005) reported that the patterns of co-morbidity occurred with such frequency that there could be an argument for the downgrading of these conditions from disorders per se to symptoms and the patterns of co-morbidity may fit the criteria for a developmental delay syndrome. Robert Melillo (2009) called these children the "Disconnected Kids”. Reduced synchronized activity between the two hemispheres is then said to materialize into one of the disorders.

The co-occurrence of these specialties may be caused by overlapping developmental pathways and interacting genetic and environmental influences that we call now atypical development, and more and more data reveals the connection of the atypical development to the giftedness (Gyarmathy, 2009). 


\subsection{Sensory motor synchronization (SMS) and spontaneous motor tempo (SMT) area}

The synchronization of one's movements to an external beat is a typically - though not exclusively human trait. There are very few other species that are able to synchronize their movements to a beat and to our current knowledge none of these come close to humans neither in precision nor in the variety of tempi in which they can achieve relative synchrony (Large, Gray, 2015). We feel an urge to follow repetitive sounds and find many associated activities rewarding (dancing, singing, playing an instrument, etc.) (Todd, Lee, 2015).

Sensory motor synchronization (SMS) is a behavior in which movements are synchronized to an external referent. In a well-studied paradigm, participants have to tap along with repetitive, predictable auditory stimuli, for example, the regular clicks of a metronome. The mean difference of the taps from the referent shows how precisely the person could adapt to the beat. For a detailed summary of the history and present findings of SMS studies see Repp (2005) and Repp \& Su (2013).

In another popular paradigm, participants are asked to tap at their preferred speed. The mean difference of the taps (ITI - Inter Tap Interval) is called spontaneous motor tempo (SMT) which is also a well-studied aspect of tempo related skills. A study of 3054 to 95-year-olds (McAuley, Jones, Holub, Johnston \& Miller, 2006) found that SMT was the highest among 4-5-year-old children (around 200 bpm) and showed decline until it reached a mean of $86 \mathrm{bpm}$ at $75 \mathrm{yrs}$. They've come to the conclusion that this slowing of tempo from childhood to the plateau period observed in adulthood is a correlate of the maturation of the nervous system. Examining SMS performance they found that the ability to synchronize to an external beat improved with age until the early 20's and also declined with old age.

Synchronizing to a beat close to one's preferred tempo was observed to be easier and tapping along in different tempos improved with aging until early adulthood. Although there is a wide consensus about the changing of SMS success across the life span (Thompson, White-Schwoch, Tierney \& Kraus, 2015) and it's relation to neural maturity, some argue that SMT is mediated by physiometric features (Moelants, 2002) so the "inner tempo" reflects the size of parts of the body involved in the complex movement of tapping.

\subsubsection{The link between rhythm and language}

A growing body of research suggests that there is a link between rhythm perception-production and phonological and reading abilities and atypical language development (Wolff, 2002; Carr, WhiteSchwoch, Tierney, Strait \& Kraus, 2014; Cumming, Wilson, Leong, Colling \& Goswami, 2015).

In a study (Wolff, 2002), dyslexic children were found to over anticipate the beat in SMS tasks by $100 \mathrm{~ms}$. They also showed greater inner inconsistency in their taps measured by ITI variability, and they were less successful in re-synchronizing after a change in tempo. Carr et al. (2014) found that 34-year-old children who were able to tap along with a steady beat of 100 and $120 \mathrm{bpm}$ showed higher performance in phonologic awareness, short-term memory, rapid naming of objects and colors, and also in rhythm and pitch discrimination tasks.

In a recent study (Cumming et al, 2015) 45 SLD children were given musical and verbal discrimination and SMS tasks. The participants were split into groups according to their intelligence. The below average IQ group showed lower performance compared to the control group in every rhythmic task, but in the normal IQ range, only their performance in the SMS task differed. In a longitudinal study of dyslexic children (Goswami et al., 2013) SMS was found to be a good predictor of the reading performance measured two years later.

There is also significant evidence, that music-based intervention programs that focus mainly on rhythm can be effective in developing reading abilities. Bhide, Power and Goswami (2013) used such a program on 6-7-year-old poor readers. Tasks included tapping with a metronome (60-140 bpm), rhythm reproduction and discrimination and tempo discrimination. A significant development was observed in the participants' reading performance which correlated with the tapping task and compared to a computer-based phoneme-grapheme intervention program, used as a control, the two had similar effects.

In a recent study (Habib et al., 2016) dyslexic children received intensive musical training: in one case during 18 hours for three consecutive days and in another, 18 hours in a 6 week period. They measured improved categorical and auditory perception in speech as a result of the first intervention 
and additional improvements in phonological awareness, auditory attention, reading abilities, and pseudoword repetition in the second case.

\subsubsection{Neural background of auditory synchronization}

The analysis of an external auditory stimulus, the extraction of the beat, the execution of a synchronized motor response and continuous monitoring and error correction involves several cortical and subcortical areas of the brain.

According to Repp and Su's analysis (2013) of recent findings of the neuroscience literature of SMS, there are possibly two distinct networks involved in tempo perception, for "automatic timing” and for "cognitively controlled timing". Former is activated by subsecond events and it is subserved by the motor system, including the cerebellum, primary and secondary motor areas and it's linked to the timing of movements. The latter involves prefrontal areas, the parietal cortex, and the basal ganglia, and it's attentionally modulated.

In a 2010 metaanalysis Wiener, Turkeltaub \& Coslett (in Repp \& Su, 2013) came to similar findings: subcortical areas, primarily the basal ganglia and the cerebellum were active in subsecond tasks, while tasks with longer intervals involved activation of the supplementary motor area and prefrontal areas. Furthermore, several studies confirm that these areas were active during both perception and production tasks. These findings may support theories that presume a significant role of the cerebellum in the emergence of specific learning difficulties (Pernet et al, 2009; Stoodley, 2015).

\subsection{Our testing area}

Todd et al. (1999) developed the sensor-motor theory of rhythm induction. Rhythm perception is a complex sensory representation of the auditory input, the sense of motion from sound which is mediated by the vestibular system, a motor representation of the body.

We have chosen the rhythm for testing area, because the sensory-motor synchronization, the neural adaptation and building the automatic processes are common in all types of atypical development, and keeping the beat needs all these functions (Repp, 2005). That way it is a perfect area to examine the differences between the results of typical and atypical developing subjects using different tools.

\section{METHODOLOGY}

Measuring the ability to synchronize to a beat could have the potential to be used as a diagnostic tool because executing simple movements to music doesn't require special musical knowledge and could possibly be tested prior to the acquisition of reading skills. In this study, we used a series of computerbased musical tasks to measure the ability of 5th-grade students of a typical elementary school and a school for SLD students.

\subsection{Tasks}

Our test consisted of 5 tasks: spontaneous motor tempo (SMT), the perception of beat asynchrony and tapping to a beat in three different tempos $(80,120,150 \mathrm{bpm})$ including constant, gradually and suddenly changing tempo. As it was designed for children from 1st to 5 th grade, our goal was to keep the test relatively short (20-25 mins) and amusing as possible to keep the participants' motivation high. From the same reason, we decided to use complex musical stimuli instead of a metronome. Live music, however, is difficult to use as a referent as the musicians themselves are sometimes slightly ahead or behind the beat. We took advantage of the flexibility of the MIDI (Musical Instrument Digital Interface) format and created the musical stimuli with virtual instruments (VST). Three instrumental versions of popular songs were used throughout the study:

Table 1. Musical stimuli used In the study. All three songs were instrumental and MIDI generated, using virtual instruments (VST)

\begin{tabular}{|l|l|}
\hline Title & Tempo \\
\hline All I have to do is dream (Everly Brothers) & $80 \mathrm{bpm}$ \\
\hline Michelle (The Beatles) & $120 \mathrm{bpm}$ \\
\hline Johnny B. Goode (Chuck Berry) & $150 \mathrm{bpm}$ \\
\hline
\end{tabular}


Task 1 - Spontaneous motor tempo: In this task, participants were asked to tap for 30 seconds at their preferred speed. Mean ITI (Inter Tap Interval) was calculated from the first and last 10 taps. The first 10 were considered the preferred tempo, while the last 10 were used to measure spontaneous acceleration and relative spread as an indicator of inner inconsistency.

Task 2 - Tempo perception: We added a metronome sound to the musical stimuli in three conditions used in the Beat Alignment Test (BAT) of Iverson and Patel (Miyazaki et al., 2008): „on the beat”, „phase error" and „tempo error”. In the first condition, the metronome sound matched the music precisely. In the second the tempo was the same, but the phase was slightly shifted, while in the third condition the tempo itself was different. Participants had to make right-wrong judgments after listening to the music.

Task 3 - Tapping to a steady beat: We used the so-called "synchronization-continuation" paradigm in this task (see Repp, 2005). The children had to synchronize their tapping to the beat of the three songs and after about 30 seconds as the music stopped, continue tapping for 20 more seconds. We calculated the relative mean difference of the taps compared to the referent, ITI variability, and also acceleration relative to the referent tempo in the continuation phase.

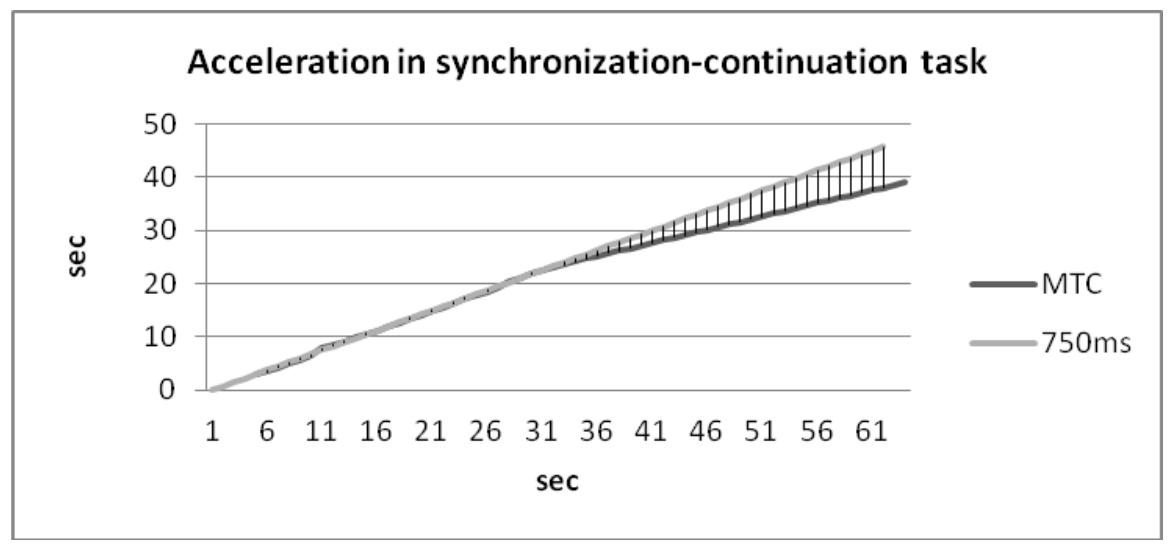

Figure 1. Acceleration observed in Task3 - MTC (MIDI Time Code) indicates the time of taps, $750 \mathrm{~ms}$ (80bpm) is the referent tempo of the music stimulus. Music stops at 21s, tempo reaches ca $125 \mathrm{bpm}$ at the end of the trial

Task 4 - Tapping to gradually changing tempo: In this tasks, the songs were manipulated to gradually change tempo during the trials. The first two were set to speed up from 80 to $105 \mathrm{bpm}$ and from 120 to $140 \mathrm{bpm}$, while third one slowed down from 150 to $135 \mathrm{bpm}$. Synchronization was calculated as the relative mean difference of the taps compared to the momentary referent tempo.

Task 5 - Sudden tempo change: In these conditions, participants experienced a sudden change in tempo after about 20 seconds and a leap back to the original tempo after 10-14 seconds (depending on the particular music). Similar to Task 4, the first two songs were sped up to 100 and $140 \mathrm{bpm}$ and the third slowed down to $120 \mathrm{bpm}$. Synchronization was calculated as in earlier tasks for all three parts of the trials: tapping to a steady tempo, altered tempo and re-synchronizing to the starting tempo. ITI variability was also measured as earlier.

\subsection{Participants}

30 children participated in the study, all of them 5th-grade students, 19 from a typical elementary school and 10 from an institution for children with specific learning difficulties (SLD school). Their mean age was 11,58 years $(s d=0,43), 18$ of them were male. Due to the lack of SLD diagnosis or previous related testing we decided to analyze their performance as a single group. Doing so we could identify 6 individuals, whose performance was below average. 2 of them were from the typical group $(10,5 \%)$ and 4 from the SLD school group (40\%). Informed consent form was gathered from all of the children's parents.

\subsection{Equipment}

We used an AKAI LPD-8 MIDI controller with rubber pads slightly raised for lighter action. The children listened to music through a Steinberg U-22 audio interface and Audio-Technica ATH-T200 closed 
headphones. Participants were allowed to set their optimal volume level and to tap in any way comfortable for them with their dominant hands. The stimuli were presented using Steinberg Cubase 5 and taps recorded in MIDI format.

\section{RESULTS}

\subsection{Beat perception}

Most of the participants were able to successfully distinguish right and wrong matches of music and metronome sounds with an overall mean performance of $77 \%(s d=1,8)$. There was a statistically significant difference between the three metronome conditions, participants encountered greater difficulty in the „phase error” variation. [ANOVA $F(2,56)=5.877(p=0.0048)^{* *}$ Tukey: T12= 3.95* $\left.\mathrm{T} 13=0.46 \mathrm{~T} 23=4.41^{* *}\right]$

Table 2. Percentage of right choices in the perception task in three conditions: "on beat", „phase error" and "tempo error"

\begin{tabular}{|c|c|c|}
\hline Condition & $\mathbf{m n}$ & sd \\
\hline "on the beat" & $82,76 \%$ & 0.688 \\
\hline "phase error" & $\mathbf{6 3 , 2 3 \% *}$ & 1.047 \\
\hline "tempo error" & $85,06 \%$ & 0.783 \\
\hline
\end{tabular}

No differences were found between sexes, and SMT indicators were also independent of perception performance. Strong correlation, however, was found with overall synchronization. [Pearson ( $r=-$ $\left.\left.0.638^{* * *} p=0.0002\right)\right]$. Children who were more successful in the perception task showed lower relative mean difference from the referent tempo in their tapping through all of the synchronization tasks. This finding seems to support the idea that perception and production are not separate abilities, they are strongly linked together as their neural background would suggest (Repp, Su, 2013).

\subsection{Spontaneous motor tempo}

We found our participants' mean SMT (103 bpm, sd=25, 83) to be somewhat lower than in the study of McAuley et al. (2006), but this difference didn't show statistical significance using one-tailed t-test $[t(28)=-1.273(p=0.2135)]$. It's also noticeable that there were large personal differences in the preferred tempo of the children with a minimum value of $51 \mathrm{bpm}$, and a maximum of $166 \mathrm{bpm}$. The variability of the taps was $14,3 \%$, and we found the acceleration of tempo to be more typical than slowing. A mean relative acceleration of $8,4 \%$ was observed $(s d=17)$, while the absolute value of tempo difference between the first and last 10 taps was $14,3 \%$.

\subsection{Synchronization performance}

Several indicators were calculated to describe the synchronization performance of the participants. There was no significant difference in different tempos. We expected that tapping to suddenly changing tempo would be the most difficult of the three conditions, which turned out to be true, but interestingly, gradually changing speed didn't cause decay (in fact, performance was slightly better) as seen in the table below. Synchronizing to a gradually changing beat possibly requires less cognitive control than adaptation to a sudden leap, so the finding that performance didn't show a significant difference in this condition compared to tapping to a steady beat may support the idea of separate neural networks underlying automatic and attention modulated timing (Repp, Su, 2013) 
Table 3. Relative difference of taps from the referent tempo in 4 conditions: steady tempo, gradually changing tempo, sudden change in tempo(1) and sudden change to the original tempo(2). Low percentage indicates more precise synchronization

\begin{tabular}{|c|c|c|}
\hline Condition & $\mathbf{m n}$ & sd \\
\hline Steady tempo & $11.90 \%$ & 0.0656 \\
\hline Gradually changing & $10.2 \%$ & 0.0472 \\
\hline Sudden change 1 & $17 \%$ & 0.0707 \\
\hline Sudden change 2 & $14,9 \%$ & 0.0539 \\
\hline \multirow{2}{*}{\multicolumn{3}{|c|}{$\begin{array}{l}\text { Kruskal-Wallis: } r F(3,84)=25.324 \quad(p=0.0000) \\
* * * \\
* \text { Tukey }(k=4, d f=84): T 12=4.10^{*} \quad T 13=6.63\end{array}$}} \\
\hline & & \\
\hline \multicolumn{3}{|l|}{ ** $T 14=5.66^{* *} T 23=10.73$} \\
\hline
\end{tabular}

The total mean relative difference from the referent tempo in all synchronization tasks was calculated as an indicator of overall adaptation performance $(m n=13,2 \% \quad s d=5,38 \%)$. A lower percentage indicates more precise adaptation in this case. No differences were found between male and female students. Using this indicator we split our sample into three groups: students with a score above mean plus one spread were called „above average”, those below mean minus one spread „below average”, while those between the „average” synchronizers.

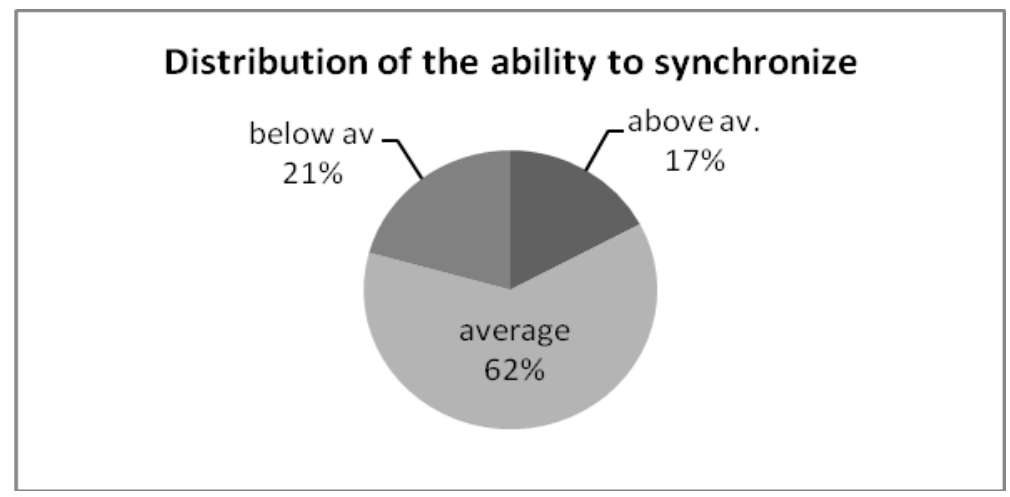

Figure 2. Distribution of "below average”, ,average” and „above average” synchronizers in the total population $(N=29)$

When comparing the two schools according to this distribution we found that $40 \%(n=4)$ of the children from the SLD school were "below average" synchronizers, while only $10,5 \%(n=2)$ from the typical school group were in this category.

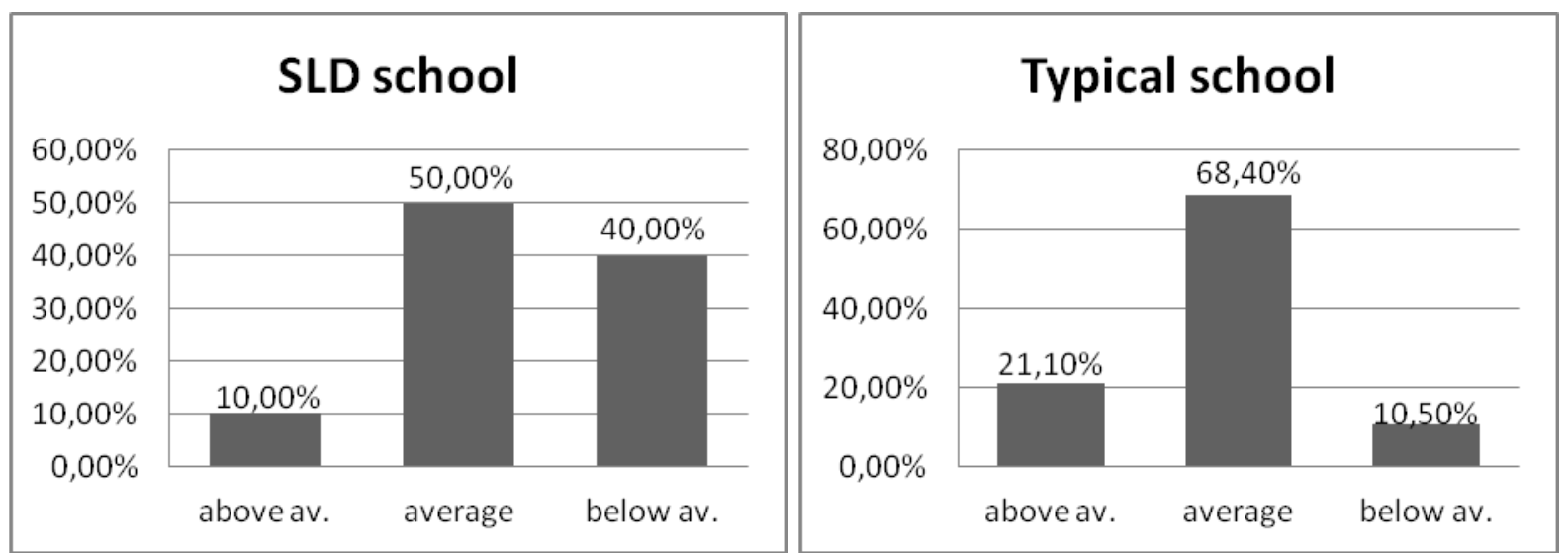

Figure 3. Percentage of „above average”, „average” and „below average” synchronizers in the SLD and typical elementary school. 


\subsection{Characteristics of „below average” synchronizers}

We examined the characteristics of the „below average” group $(n=6)$ and found several differences compared to other students. They were significantly less successful in the perception task. Their overall ratio of right answers was 59,3\% while the rest of the population reached $81,8 \%$ [Two-tailed $t$ test $\left.t(27)=3.042(p=0.0052)^{* *}\right]$. We also found that this difference only emerged in „on the beat” condition in which the ratios were 61,2 and $88,5 \%$, similarly with the "below average" group's disadvantage. They didn't, however, differ in their performance when different musical stimuli were compared.

In the SMT task students from this group showed no difference from others in preferred tempo or ITI variability. Relative acceleration (both positive and negative) was found to be higher $(15,7 \%)$ in the "below average" group than in the rest of the students $(9,34 \%)$ but with statistical significance slightly below the $5 \%$ margin [Welch: $d(25.4)=1.926(p=0.0654)+]$. The lack of significant difference in ITI variability shows that even the least successful synchronizers were able to tap a relatively steady beat in at their preferred speed.

The "below average" group showed higher ITI variability in two subtasks: the steady tempo condition of Task3 when tapping to slow (80 bpm) and fast tempo (150 bpm) music.

Table 4. ITI variability of tapping in the "below average" group compared to the other students

\begin{tabular}{|c|c|c|c|}
\hline \multicolumn{4}{|c|}{ ITI (Inter Tap Interval) variability } \\
\hline Subtask & $\begin{array}{c}\text { "Average" and } \\
\text { "Above } \\
\text { average" group }\end{array}$ & $\begin{array}{c}\text { "Below } \\
\text { average" group }\end{array}$ & Statistical test \\
\hline Steady $80 \mathrm{bpm}$ & $5.72 \%$ & $8.38 \%$ & $\mathrm{t}(27)=-2.807(\mathrm{p}=0.0092)^{\star *}$ \\
\hline Steady 150 bpm & $6.91 \%$ & $16.90 \%$ & $\mathrm{~d}(5.3)=-2.679 \quad(\mathrm{p}=0.0416)^{\star}$ \\
\hline
\end{tabular}

We uncovered significant differences in negative mean asynchrony (NMA). The "below average" synchronizers were more likely to over-anticipate the beat. This was observable in the case of the slow musical stimuli (steady tempo condition in Task3 \& Task5), and the mid-tempo music (steady condition in Task3, gradually changing condition in Task4, and suddenly changing tempo condition in Task5). This finding is noteworthy as NMA is thought to diminish or disappear in complex rhythmical context or in the presence of subdivision (Repp, 2005).

Table 5. NMA (negative mean asynchrony) in steady beat subtasks. Larger over-anticipation was found in the "below average" group

\begin{tabular}{|c|c|c|c|c|c|}
\hline \multicolumn{7}{|c|}{ Over-anticipation (NMA) } & \\
\hline & $\begin{array}{c}\text { "Average } \\
\text { " and } \\
\text { "Above } \\
\text { average" } \\
\text { group }\end{array}$ & $\begin{array}{c}\text { "Below } \\
\text { average" } \\
\text { group }\end{array}$ & $\begin{array}{c}\text { Differe } \\
\text { nce (\%) }\end{array}$ & $\begin{array}{c}\text { Differenc } \\
\text { e (ms) }\end{array}$ & Statistical test \\
\hline 80 bpm (Task3, steady cond.) & $-4.67 \%$ & $-11.70 \%$ & $-7.03 \%$ & -52.70 & $\begin{array}{c}\mathrm{t}(27)=2.460 \\
(\mathrm{p}=0.0206)^{*}\end{array}$ \\
\hline 80 bpm (Task5, steady cond.) & $-4.8 \%$ & $-13.00 \%$ & $-8.2 \%$ & -61.50 & $\begin{array}{c}\mathrm{d}(8.0)=3.202 \\
(\mathrm{p}=0.0127)^{*}\end{array}$ \\
\hline 120 bpm (Task 3, steady cond.) & $-7.78 \%$ & $-23.10 \%$ & $-15.32 \%$ & -76.60 & $\begin{array}{c}\mathrm{d}(12.9)=5.918 \\
(\mathrm{p}=0.0001)^{* * *}\end{array}$ \\
\hline $120-140$ bpm (Task4, gradually \\
changing cond.) & $-1.67 \%$ & $-13.20 \%$ & $-11.53 \%$ & -57.65 & $\begin{array}{c}\mathrm{t}(27)=4.389 \\
(\mathrm{p}=0.0002)^{* * *}\end{array}$ \\
\hline $\begin{array}{c}140 \text { bpm (Task5, suddenly } \\
\text { changing cond.) }\end{array}$ & $-1.80 \%$ & $-11.00 \%$ & $-9.20 \%$ & -69.00 & $\begin{array}{c}\mathrm{t}(27)=2.422 \\
(\mathrm{p}=0.0224)^{*}\end{array}$ \\
\hline
\end{tabular}




\section{CONCLUSIONS}

Using our test we were able to measure performance in tempo-related rhythmical tasks with the help of only complex musical stimuli. Performance in the perception and the synchronization task showed a strong correlation, which suggests that these two domains are indeed linked together. We could identify a group of "below average" synchronizers, whose taps showed greater difference from the referent, and also inner inconsistency. They were also characterized by poor perception of tempo and a larger tendency to over-anticipate the beat when tapping along with the music. When comparing the two populations, we found that $40 \%$ of the students from the SLD school were "below average" synchronizers, while this ratio was only $10.5 \%$ in the "typical school" group.

The higher percentage of the "below average" synchronizers among the SLD students shows that the method definitely can capture a type of neurological deviance. However there were "below average" synchronizers among the "typical" students, too, though the ratio was only about the quarter of the SLD group's poor synchronizer ratio. Plus, there were good synchronizers among the SLD students, too. The matching is far not unambiguous. Certainly the diagnoses of some students with SLD could be false, and there may be some SLD students in the "typical" class, too. If that is the case, and a thorough assessment could identify SLD in those "typical" students who achieved poorly in our measure and reveal other causes than SLD behind the learning difficulties of the students who achieved well in our task but have SLD diagnoses, our method could be considered a perfect identifier.

Further studies should bring light to the role of other factors in the connection of SLD and beatkeeping, and to the fine relationship between beat-keeping and cognitive and academic performance. To make the investigation more extensive, the next phase of our research aims at migrating the test process to mobile environment. A tablet device can be used to control the subtasks, and its touchscreen provides a way to record the physical hits of the user as touch events. An open question is whether the hard glass surface of the screen influences the results of the auditive tests.

Another direction is to test the beat keeping ability with other modalities. Namely, visual and kinesthetic tests are planned to gather statistics from subjects with inferior auditive skills, and to compare the beat keeping ability in different situations

\section{ACKNOWLEDGEMENTS}

The authors wish to thank the students of Németh Imre and Meixner Elementary School, who participated in the study and their teachers for their kind support: Ildikó Burján, Ádám Fasimon, Bea Gombár, Viktória Kriston, Zsóka Sipos, Béla Sum, Márta Tiborcz, Ágnes Ungár and Nóra Veinelt.

\section{REFERENCES}

[1] R. Barkley, "Attention-deficit/hyperactivity disorder, selfregulation, and time: Toward a more comprehensive theory." Journal of Developmental and Behavioral Pediatrics, 18, 271-279, 1997.

[2] A. Bhide, A. Power, U. Goswami, "A rhythmic musical intervention for poor readers: A comparison of efficacy with a letter-based intervention. Mind, Brain, and Education, 7(2), 113123, 2013.

[3] K. W. Carr, T. White-Schwoch, A. T. Tierney, D. L. Strait, N. Kraus, "Beat synchronization predicts neural speech encoding and reading readiness in preschoolers." Proceedings of the National Academy of Sciences, 111(40), 14559-14564, 2014

[4] R. Cumming, A. Wilson, V. Leong, L. J. Colling, U. Goswami, "Awareness of rhythm patterns in speech and music in children with specific language impairments." Frontiers in human neuroscience, 9, 2015.

[5] M. B. Denckla, R. G. Rudel, C. Chapman, J. Krieger, "Motor proficiency in dyslexic children with and without attentional disorders. Archives of Neurology, 42, 228-231, 1985.

[6] S. I. Greenspan, S. Wieder, "A functional developmental approach to autism spectrum disorders." Journal of the Association for Persons With Severe Handicaps, 24, 147-161, 1999. 
[7] V. Gross-Tsur, O. Manor, R. S. Shalev, "Developmental dyscalculia: Prevalence and demographic features." Developmental Medicine and Child Neurology, 38, 25-33, 1996.

[8] E. Gyarmathy, "Atypical brain and the gifted I. - Giftedness and the neurologically based achievement difficulties and the Asperger syndrome" Pszichológia. Vol. 29, 4. 377-390, 2009.

[9] M. Habib "The neurological basis of developmental dyslexia: An overview and working hypothesis" Brain, 123 (12): 2373-2399, 2000.

[10] M. Habib, C. Lardy, T. Desiles, C. Commeiras, J. Chobert, M. Besson "Music and dyslexia: a new musical training method to improve reading and related disorders." Frontiers in psychology, 7, 2016.

[11] E. L. Hill, "Executive dysfunction in autism" Trends Cogn. Sci. 2004 Jan;8(1):26-32, 2004.

[12] Cs. Kertesz (2016) "Tempo észlelés és szenzomotoros szinkronizáció számítógépes vizsgálata.” Budapest, Károli Gáspár Református Egyetem, 2016.

[13] E. W. Large, P. M. Gray, "Spontaneous tempo and rhythmic entrainment in a bonobo (Pan paniscus)." Journal of Comparative Psychology, 129(4), 317, 2015.

[14] S. D. Mayes, S. L. Calhoun, "Frequency of reading, math and writing disabilities in children with clinical disorders." Learning and Individual Differences, 16, 145-157, 2006.

[15] J. D. McAuley, M. R. Jones, S. Holub, H. M. Johnston, N. S. Miller, "The time of our lives: life span development of timing and event tracking." Journal of Experimental Psychology: General, 135(3), 348, 2006.

[16] R. Melillo "Disconnected Kids: The Groundbreaking Brain Balance Program for Children with Autism, ADHD, Dyslexia, and Other Neurological Disorders.” New York: Perigee Press. 2009.

[17] D. Patel, J R. Iversen, "The Beat Alignment Test (BAT): Surveying beat processing abilities in the general population", In. (Eds) K. I. Miyazaki, Y. Hiraga, M. Adachi, Y. Nakajima, M. Tsuzaki: Proceedings of the $10^{\text {th }}$ International Conference on Music Perception and Cognition (ICMPC 10) Sapporo, Japan. 465-468, 2008.

[18] D. Moelants, "Preferred tempo reconsidered." In Proceedings of the 7th international conference on music perception and cognition . Vol. 2002, 1-4, 2002, July.

[19] R. Pauc, "Comorbidity of dyslexia, dyspraxia, attention deficit disorder (ADD), attention deficit hyperactive disorder (ADHD), obsessive compulsive disorder (OCD) and Tourette's syndrome in children: A prospective epidemiological study. Clinical Chiropractic, Volume 8 , Issue 4, December, 189-198 2005.

[20] C. R. Pernet, J. B. Poline, J. F. Demonet, G. A. Rousselet, "Brain classification reveals the right cerebellum as the best biomarker of dyslexia." BMC neuroscience, 10(1), 67, 2009.

[21] J. P. Piek, T. Pitcher, D. A. Hay, "Motor coordination and kinaesthesis in boys with attention deficit-hyperactivity disorder." Developmental Medicine and Child Neurology, 41, 159-165, 1999.

[22] A., Reiter, O. Tucha, K. W. Lange, "Executive functions in children with dyslexia." Dyslexia, Volume 11, Number 2, 1 May. (16) 116-131, 2005.

[23] B. H. Repp, "Sensorimotor synchronization: a review of the tapping literature." Psychonomic bulletin \& review, 12(6), 969-992, 2005.

[24] B. H. Repp, Y. H. Su, "Sensorimotor synchronization: a review of recent research (2006-2012)." Psychonomic bulletin \& review, 20(3), 403-452, 2013.

[25] I. Schonfeld, D. Shaffer, J. Barmack, "Neurological soft signs and school achievement: The mediating effects of sustained attention." Journal of Abnormal Child Psychology, 17, 575-596, 1989.

[26] J. A. Sergeant, H. Geurts, J. Oosterlaan, "How specific is a deficit of executive functioning for Attention-Deficit/Hyperactivity Disorder?" Behav. Brain Research Volume 130, Issues 1-2, 10 March 3-28, 2002. 
[27] R. J. Shaffer, L. E. Jacokes, J. F. Cassily, S. I. Greenspan, R. F. Tuchman, P. J. Stemmer, Jr. "Effect of Interactive Metronome ${ }^{\circledR}$ training on children with ADHD. American Journal of Occupational Therapy, 55, 155-162, 2001.

[28] S. E. Shaywitz, B. E. Shaywitz, "Attention deficit disorder: Current perspectives." In J. F. Kavanaugh, T. J. Truss (Eds.), Learning disabilities: Proceedings of the national conference Parkton, MD: York Press. 369-523, 1988.

[29] C. J. Stoodley, "The cerebellum and neurodevelopmental disorders." The Cerebellum, 15(1), 34-37, 2016.

[30] E. C. Thompson, T. White-Schwoch, A. Tierney, N. Kraus, "Beat synchronization across the lifespan: intersection of development and musical experience." PloS one, 10(6), e0128839, 2015.

[31] N. P. Todd, C. S. Lee, "The sensory-motor theory of rhythm and beat induction 20 years on: a new synthesis and future perspectives." Frontiers in human neuroscience, 9, 444, 2015.

[32] N.P.M. Todd, D.J. O'Boyle, C.S. Lee A sensory-motor theory of rhythm, time perception and beat induction. J. New Music Research. 28, 5-29, 1999.

[33] M. C. Welsh, B. F. Pennington, D. B. Groisserc, "A normative-developmental study of executive function: a window on prefrontal function in children." Dev. Neuropsychol. 7, 131-149, 1991.

[34] P. H. Wolff, "Timing precision and rhythm in developmental dyslexia. Reading and Writing, 15(12), 179-206, 2002. 